\title{
Longitudinal Restriction Spectrum Imaging Is Resistant to Pseudoresponse in Patients with High-Grade Gliomas Treated with Bevacizumab
}

P.D. Kothari, N.S. White, N. Farid, R. Chung, J.M. Kuperman, H.M. Girard, A. Shankaranarayanan, S. Kesari, C.R. McDonald, and A.M. Dale

\begin{abstract}
BACKGROUND AND PURPOSE: Antiangiogenic therapies, such as bevacizumab, decrease contrast enhancement and FLAIR hyperintensity in patients with high-grade gliomas in a manner that may not correlate with actual tumor response. This study evaluated the ability of an advanced DWI technique, restriction spectrum imaging, to improve conspicuity within regions of restricted diffusion compared with $A D C$ in patients treated with bevacizumab and to demonstrate that unlike ADC, restriction spectrum imaging is less affected by bevacizumab-induced reductions in FLAIR hyperintensity.
\end{abstract}

MATERIALS AND METHODS: Restriction spectrum imaging cellularity maps and DWI were available for 12 patients with recurrent high-grade gliomas at baseline and following initiation of bevacizumab. VOls were drawn for regions of restricted diffusion, surrounding FLAIR hyperintensity, and normal-appearing white matter; and intensity values within regions of restricted diffusion and FLAIR hyperintensity were normalized to normal-appearing white matter. Normalized values were compared between restriction spectrum imaging cellularity maps and ADC at baseline and on treatment by using repeated-measures ANOVA.

RESULTS: All patients exhibited decreases in contrast enhancement and FLAIR hyperintensity following treatment. Normalized intensity values were higher on restriction spectrum imaging cellularity maps compared with ADC in regions of restricted diffusion, whereas intensity values were higher on ADC compared with restriction spectrum imaging cellularity maps in regions of FLAIR hyperintensity. Bevacizumab-induced decreases in FLAIR hyperintensity had a greater effect on ADC than on the restriction spectrum imaging cellularity maps, with the relative sensitivity of ADC to changes in FLAIR hyperintensity being $>20$ times higher than that on restriction spectrum imaging cellularity maps.

CONCLUSIONS: Restriction spectrum imaging is less influenced by reductions in FLAIR hyperintensity compared with ADC, which may confer an advantage of restriction spectrum imaging over ADC for interpreting tumor response on imaging following antiangiogenic therapy.

ABBREVIATIONS: FLAIR-HI = FLAIR hyperintensity; GBM = glioblastoma multiforme; NAWM = normal-appearing white matter; RD = restricted diffusion; RSI-CMs $=$ restriction spectrum imaging cellularity maps

$\mathrm{C}$

urrent radiographic criteria for monitoring patients with high-grade glioma are heavily dependent on changes in contrast enhancement and FLAIR signal on standard MR images. ${ }^{1}$ However, with greater use of antiangiogenic treatments such as

Received October 12, 2012; accepted after revision December 16.

From the School of Medicine (P.D.K.); Departments of Radiology (N.S.W., N.F., R.C., J.M.K., A.M.D.), Neurosciences (S.K., A.M.D.), and Psychiatry (C.R.M., A.M.D.); Multimoda Imaging Laboratory (N.S.W., N.F., J.M.K., H.M.G., C.R.M., A.M.D.); and Translational Neuro-Oncology Laboratories (S.K.), Moores Cancer Center; University of California, San Diego, La Jolla, California; and GE Healthcare (A.S.), Milwaukee, Wisconsin. Drs McDonald and Dale contributed equally as senior authors.

This work was supported by NIH grants R01AG03122 (A.M.D.), R01EB000790 (A.M.D.), RC2DA029475 (A.M.D.), EB000790 (A.M.D.), 3P30CA023100-25S8 (S.K.), TL1 TR000098-03 (P.D.K.), and R01NS065838 (C.R.M.).

None of the authors have any personal or institutional financial interest in drugs, materials, or devices described in this submission. bevacizumab, an anti vascular endothelial growth factor antibody, using contrast enhancement as a surrogate for tumor response has become increasingly challenging. ${ }^{2}$ Patients with recurrent highgrade glioma treated with bevacizumab often exhibit a sharp decrease in contrast enhancement and edema without a corresponding clinical response-a phenomenon called "pseudoresponse"- due to the ability of antiangiogenic agents to normalize hyperpermeable tumor vasculature, thus restoring the blood-brain barrier., ${ }^{3,4}$

DWI and ADC have been proposed as imaging markers for tumor response in the presence of antiangiogenic agents to ad-

\footnotetext{
Please address correspondence to C.R. McDonald, MD, Multimodal Imaging Laboratory, Ste C101, 8950 Villa La Jolla Dr, La Jolla, CA 92037; e-mail: camcdonald@ucsd.edu

- Indicates open access to non-subscribers at www.ajnr.org

http://dx.doi.org/10.3174/ajnr.A3506
} 


\begin{tabular}{|c|c|c|c|c|c|c|}
\hline Patient & $\begin{array}{l}\text { Age }(y r) / \\
\text { Sex }\end{array}$ & Pathology & Initial Therapy & $\begin{array}{l}\text { Surgery Prior to } \\
\text { Bevacizumab } \\
\text { Scan (mo) }\end{array}$ & $\begin{array}{l}\text { Pre and Post- } \\
\text { Bevacizumab } \\
\text { Scans (day) }\end{array}$ & $\begin{array}{l}\text { Post-Bevacizumab } \\
\text { Pathologyc }\end{array}$ \\
\hline 1 & $58 / F$ & GBM WHO IV & XRT + TMZ; TMZ 5/28 & GTR, 1 & $-31,+29$ & - \\
\hline 2 & $43 / M$ & AGN/WHO III/IV & XRT + TMZ; TMZ 3/14 & GTR, 12 & $-22,+16$ & AA/WHO III/IV \\
\hline 3 & $62 / F$ & GBM / WHO IV & XRT + TMZ; TMZ 3/14 & STR, 1+ & $-3,+112$ & GBM/WHO IV \\
\hline 4 & $49 / M$ & GBM/WHO IV & XRT + TMZ; TMZ 3/14 & STR, 1 & $-10,+22$ & - \\
\hline 5 & $57 / F$ & GBM/WHO IV & $\begin{array}{l}\text { XRT + TMZ; TMZ 5/28, } \\
\text { TMZ 3/14 }\end{array}$ & STR, 6+ & $-13,+49$ & GBM/WHO IV \\
\hline 6 & $63 / F$ & GBM/WHO IV & XRT + TMZ; TMZ 5/28 & STR, 1 & $-28,+46$ & $\begin{array}{l}\text { GBM/WHO IV + } \\
\text { radiation effect }\end{array}$ \\
\hline 7 & $66 / M$ & GBM/WHO IV & XRT + TMZ; TMZ 5/28 & STR, 5 & $-2,+34$ & $\begin{array}{l}\text { GBM/WHO IV + } \\
\text { radiation necrosis }\end{array}$ \\
\hline 8 & $56 / M$ & AOA/WHO III & XRT + TMZ; TMZ 5/28 & $\mathrm{RXN}, 4+$ & -15 days, +15 & - \\
\hline 9 & $67 / M$ & GBM/WHO IV & XRT + TMZ; TMZ 5/28 & GTR, 7 & $-7,+29$ days & - \\
\hline 10 & $27 / F$ & AA/WHO III & $\mathrm{XRT}+\mathrm{TMZ}$ & $\mathrm{Bx}, 2+$ & $-8,+16$ & - \\
\hline 11 & $40 / M$ & GBM/WHO IV & XRT + TMZ; TMZ 5/28 & STR, $5+$ & $-37,+35$ & - \\
\hline 12 & $56 / M$ & GBM/WHO IV & XRT + TMZ; TMZ 5/28 & STR, 7 & $-49,+27$ & - \\
\hline
\end{tabular}

Note:- AOA indicates anaplastic oligoastrocytoma; AA, anaplastic astrocytoma; XRT + TMZ, radiotherapy plus adjuvant temozolomide; TMZ 5/28, temozolomide for 5 days every 28 days; TMZ 3/14, temozolomide for 3 days every 14 days; GTR, gross total resection; STR, subtotal resection; RXN, craniotomy and resection; Bx, biopsy; AGN, anaplastic glioneural neoplasm; $\mathrm{WHO}=$ World Health Organization.

${ }^{a}$ Months shown indicate the interval between the surgical event and the first scan obtained during bevacizumab treatment.

${ }^{b}$ Interval in days between the scan prior to bevacizumab treatment (pre-bevacizumab scan), and initiation of bevacizumab therapy, followed by the interval in days between initiation of bevacizumab therapy and first scan during bevacizumab treatment (post-bevacizumab scan).

c Pathology after bevacizumab therapy had started.

dress this issue of pseudoresponse. ${ }^{5-8}$ Regions of increased cellularity, such as tumor, restrict water diffusion and decrease ADC values relative to surrounding tissue. ${ }^{9,10}$ However, concomitant edema and tumor-related necrosis increase ADC values, thereby directly opposing the reduction in ADC associated with tumor. ${ }^{11,12}$ This offset may result in diminished conspicuity of tumor on ADC maps and difficulty distinguishing tumor from NAWM. In the setting of antiangiogenic treatments, which can significantly decrease edema, the interpretation of ADC values is further complicated. ${ }^{13,14}$

To maximize sensitivity to the restricted diffusion signal within tumor cells while excluding the hindered diffusion signal associated with edema, we apply a new, advanced DWI technique called "restriction spectrum imaging." ${ }^{15}$ Whereas ADC reflects both hindered and restricted diffusion pools within a voxel, RSI uses multiple b-values and diffusion times to separate out the spherically restricted water compartment from the hindered water compartment, and it has been previously shown to improve tumor conspicuity relative to high-b-value ADC in patients with primary and metastatic brain tumors. ${ }^{16}$ Therefore, RSI may provide a more sensitive and robust biomarker of cellularity compared with conventional DWI and ADC in the context of antiangiogenic treatments that result in significant decreases in edema.

In this study, we evaluated the ability of RSI to improve conspicuity within regions of RD compared with standard ADC in patients with recurrent high-grade glioma treated with bevacizumab. In line with our previous work, we hypothesized that RSI would improve conspicuity within regions of RD over standard ADC, whereas ADC values within regions of RD would approximate those in NAWM. Moreover, we predicted that RSI would be minimally affected by bevacizumab-induced reductions in FLAIR-HI, whereas changes in ADC would be highly influenced by treatment-related reductions in FLAIR-HI due to the sensitivity of ADC to fast hindered diffusion.

\section{MATERIALS AND METHODS Patients}

All patients included in the study signed consent forms approved by the institutional review board. From December 2010 to August 2012, one hundred ninety-three patients with primary and metastatic brain tumors at the UCSD Moores Cancer Center underwent a standardized MR imaging protocol that included the RSI sequence. From this group, 12 patients met the following inclusion criteria to form the final study cohort: 1) recurrent pathologically confirmed high-grade glioma (World Health Organization grade III or IV); 2) at least 1 baseline RSI scan before initiating bevacizumab therapy and a follow-up RSI scan while on bevacizumab; and 3) no significant blood products on susceptibility-weighted images that would confound diffusion signals on the ADC or RSI scans. The average age of the final cohort was 54 years, ranging from 27 to 67 years, with 7 men and 5 women. Nine patients had GBMs, while 3 had grade III gliomas (Table). To further avoid contamination from immediate postsurgical effects such as hemorrhage and cytotoxic edema, we did not perform MRI within the first 2 months following surgery.

\section{MR Imaging}

MR imaging was performed on a 3T Signa Excite HDx scanner (GE Healthcare, Milwaukee, Wisconsin) equipped with an 8-channel head coil. Our imaging protocol included pre- and postgadolinium $3 \mathrm{D}$ volumetric $\mathrm{T} 1$-weighted inversion recovery spoiled gradient-recalled sequences $\left(\mathrm{TE} / \mathrm{TR}=2.8 / 6.5 \mathrm{~ms}, \mathrm{TI}=450 \mathrm{~ms}\right.$, flip angle $=8^{\circ}$, $\mathrm{FOV}=24 \mathrm{~cm}$, matrix $=0.93 \times 0.93 \times 1.2 \mathrm{~mm})$ and a $3 \mathrm{D} \mathrm{T} 2-$ weighted FLAIR sequence $(\mathrm{TE} / \mathrm{TR}=126 / 6000 \mathrm{~ms}$, TI $=1863 \mathrm{~ms}$, FOV $=24 \mathrm{~cm}$, matrix $=0.93 \times .093 \times 1.2 \mathrm{~mm}$ ). For RSI, a singleshot pulsed gradient spin-echo EPI sequence was used (TE/TR $=96$ $\mathrm{ms} / 17$ seconds, $\mathrm{FOV}=24 \mathrm{~cm}$, matrix $=96 \times 96 \times 48)$ with 4 b-values $\left(b=0,500,1500\right.$, and $\left.4000 \mathrm{~s} / \mathrm{mm}^{2}\right)$ with 6,6 , and 15 unique diffusion directions for each nonzero b-value, respectively (28 total volumes, $\sim 8$-minute scanning time).

AJNR Am J Neuroradiol 34:1752-57 Sep 2013 www.ajnr.org 1753 


\section{Preprocessing, ADC Calculation, and RSI Analysis}

Before analysis, raw RSI data were corrected for geometric distortions due to susceptibility, gradient nonlinearities, and eddy currents. ${ }^{17}$ This was followed by correction of patient motion by using in-house software. ADC values were calculated from a tensor fit to the full dataset (all b-values and diffusion directions).

Details of the RSI model and analysis are given in White et al. ${ }^{16}$ Briefly, the diffusion signal in each voxel was fit by using a linear mixture model of restricted and hindered water compartments with spheric and cylindric geometries. For this article, the resulting spherically restricted water fraction formed the basis of our RSI cellularity maps. To maximize sensitivity and specificity to the spherically restricted water fraction, a beam-forming filter was applied to our RSI-CMs to reduce residual signal contamination from cylindrically restricted and hindered water compartments. ${ }^{16}$

\section{Longitudinal Registration}

For longitudinal measurement of RSI-CMs and ADC in defined regions of FLAIR-HI and NAWM, it was necessary to account for the changes in brain shape caused by tumor growth or surgical resection. A nonlinear registration, as described in $\mathrm{McDonald}$ et al, ${ }^{18}$ was used to define a parameterized deformation between the first and second time points (before and after initiation of bevacizumab therapy). The T1-weighted precontrast images, weighted by T2-weighted FLAIR to correct for regions of hypointensity, were used to guide the nonlinear registration, and the computed deformations were then applied to the coregistered RSI-CM and ADC volumes.

\section{Region-of-Interest and Imaging Analysis}

Volumetric ROIs were drawn manually on baseline (pre-bevacizumab) scans for the contrast-enhancing region, the FLAIR-HI region surrounding the contrast-enhancing region, and NAWM by using coregistered 3D T1 postcontrast and 3D FLAIR volumes with the Amira software package (Visage Imaging, San Diego, California). NAWM was drawn in isointense white matter contralateral to the tumor on the 3D FLAIR sequence. All manual ROIs were approved by a board-certified neuroradiologist with 3 years of experience before image analysis. An automated region of interest was also generated for the region of $\mathrm{RD}$ within the region of contrast enhancement, and this region of interest was used to approximate the actual area of viable tumor within the contrastenhancing region. ${ }^{19}$ Of note, this $\mathrm{RD}$ region of interest was generated from the RSI sequence because our prior work has shown improved tumor conspicuity on RSI-CMs compared with ADC. ${ }^{16}$ In addition, our prior work has demonstrated that $\mathrm{ADC}$ tends to underestimate the area of $\mathrm{RD}$, whereas RSI-CMs are more inclusive of the total area of RD.

Determination of relevant ADC and RSI-CM values was performed in a manner similar to methods described by Gerstner et al. ${ }^{20}$ Each patient's baseline (pre-bevacizumab) and follow-up (on bevacizumab) scans were used for analysis. The baseline FLAIR region of interest was coregistered with the RSI-CMs and ADC maps from each scan date. This coregistration allowed determination of ADC and RSI-CM values within the region of FLAIR-HI at baseline and determination of ADC and RSI-CM values within the region of RD at baseline.

\section{Statistical Analysis}

Intensity values within the RSI-CMs and ADC maps were transformed into $z$ scores on the basis of the mean-intensity values within contralateral NAWM [(Pixel Intensity of RSI-CMs or ADC - Mean Intensity in Contralateral NAWM) / SD in Contralateral NAWM]. The $z$ scores provided a measure of the relative sensitivity of each technique to RD and FLAIR-HI, normalized to NAWM. Z scores were used in all subsequent analyses. Repeated measures ANOVAs were performed within regions of RD and FLAIR-HI with method (RSI-CMs versus ADC) and treatment (baseline versus on treatment) as repeating factors. This procedure allowed us to evaluate the relative sensitivity of each method to RD and FLAIR-HI (main effect of method) and to evaluate whether the relative sensitivity of each method to $\mathrm{RD}$ and FLAIR-HI varies as a function of treatment with bevacizumab (method by treatment interaction). The relative sensitivity to change in FLAIR-HI versus RD was also computed for each measure and was defined as follows: Mean $z$ Score Change in FLAIR-HI / Mean z Score in the Region of RD.

\section{RESULTS}

In all 12 patients who met the inclusion criteria, a visible decrease in both contrast enhancement and FLAIR-HI was observed following initiation of bevacizumab therapy. Eight patients showed a decrease of $\geq 50 \%$ in $\mathrm{T} 1$ contrast enhancement, whereas the remaining 4 patients showed a decrease of $25 \%-50 \%$. Similarly, 2 of the patients showed a corresponding decrease of $\geq 50 \%$ in FLAIR-HI surrounding the enhancing region, whereas all 12 patients showed a decrease of at least $25 \%$ in FLAIR-HI following treatment with bevacizumab. Figure 1 shows a sample patient at baseline and on treatment with bevacizumab. The decrease in T1 enhancement and FLAIR-HI can be readily appreciated in this patient following initiation of treatment. Areas of RD are more conspicuous on RSI-CMs relative to ADC both at baseline and following treatment, whereas ADC signal intensity following treatment (ie, when not surrounded by FLAIR-HI) appears similar to that in NAWM. Furthermore, the large region of decreased signal intensity on ADC following treatment appears to mirror the decreased signal intensity on the FLAIR image (Fig $1 J,-K$ ), whereas RSI-CM intensity changes do not appear similar to decreases in FLAIR hyperintensity (Fig $1 K,-L$ ).

Figure 2 shows the normalized values (ie, $z$ scores) for RSICMs and ADC in regions of RD and FLAIR-HI before and on treatment with bevacizumab averaged across patients. The repeated measures ANOVA for RD revealed a main effect of method $[F(1,11)=18.97, P<.01]$, indicating that $\mathrm{RD}$ intensity values were higher on RSI-CMs relative to ADC both before and on treatment. In fact, mean RSI-CM intensity values were approximately 8 times greater than those in NAWM before and on treatment, whereas mean ADC values were only 0.47 and 1.2 times greater than NAWM at baseline and on treatment, respectively. The repeated measures ANOVA within the region of FLAIR-HI revealed a main effect of method, indicating that FLAIR-HI values were higher on ADC relative to RSI-CMs $[F(1,11)=88.8, P<$ $.001]$. However, this was qualified by a method by treatment interaction $[F(1,11)=61.1, P<.001]$, which revealed that decreases in FLAIR-HI that occurred on treatment with bevaci- 


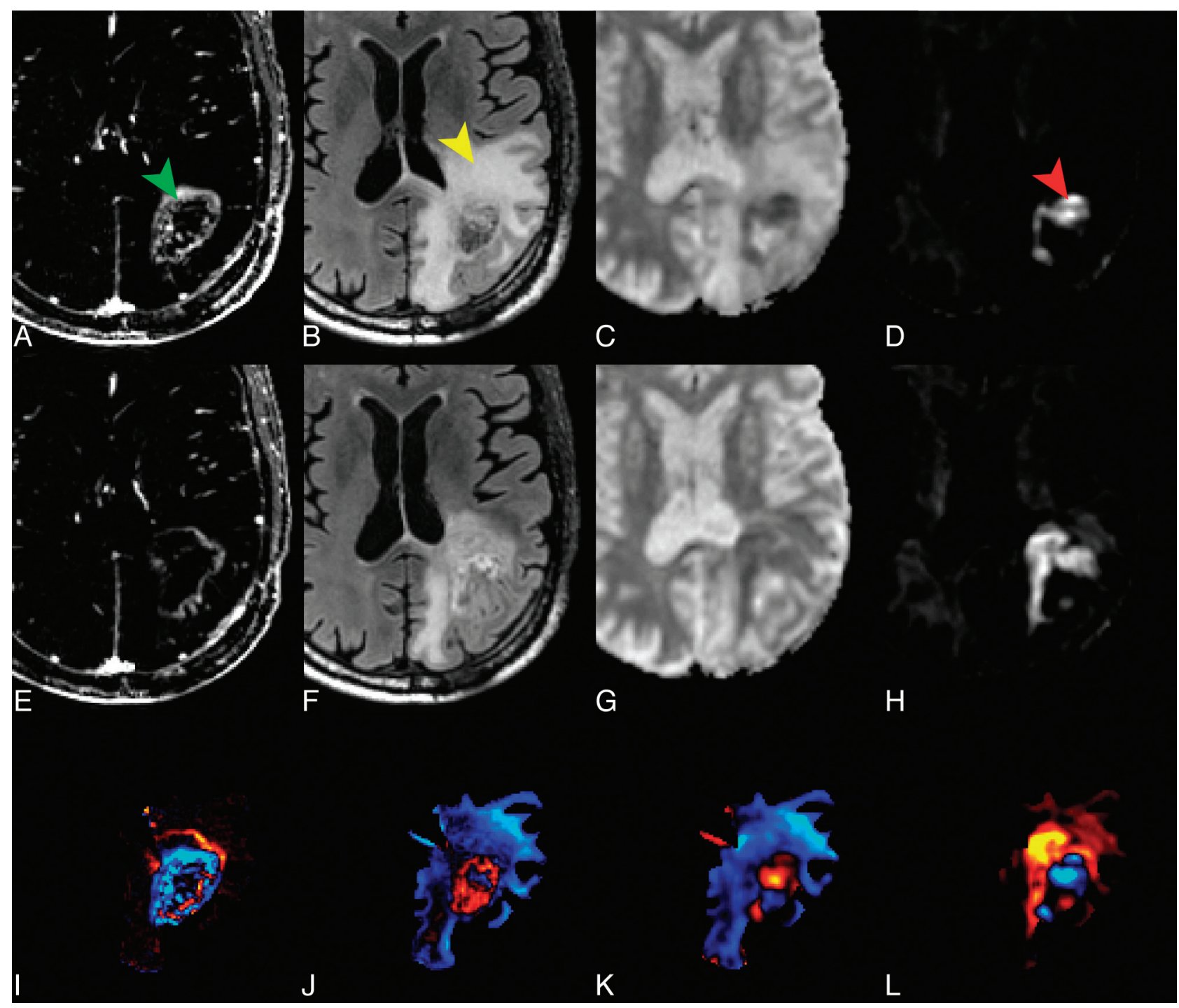

FIG 1. A 67-year-old man with left parietal GBM status after resection and chemoradiation. Top row shows the $\Pi 7$ postcontrast- $\Pi$ precontrast $(A)$, FLAIR $(B), A D C(C)$, and RSI-CM $(D)$ images before the start of bevacizumab, while the middle row shows the TI postcontrast-T precontrast (E), FLAIR $(F), A D C(G)$, and RSI-CM $(H)$ images after the initiation of bevacizumab. Arrowheads indicate the contrast-enhancing region (green), the surrounding region of FLAIR-HI (yellow), and the region of RD on RSI-CMs (red). Although there is a decrease in contrast enhancement and surrounding FLAIR-HI after the initiation of bevacizumab, the region of RD increases and becomes more confluent; this change suggests worsening residual/recurrent tumor. Moreover, this increase in the region of RD is much more conspicuous on RSI-CMs compared with the ADC. The bottom row depicts these changes on "change maps" (change in TI postcontrast-precontrast) (I), change in FLAIR (I), change in ADC (K), and change in the RSI-CMs (L), with red-yellow indicating an increase in signal intensity and blue-cyan indicating a decrease in signal intensity. Note that on the ADC change map (K), the area of increased RD is essentially masked by the decreased signal intensity within the region of surrounding FLAIR-HI.

zumab had a greater effect on ADC than on RSI-CM intensity values. As shown in Fig 3, the change in ADC following initiation of bevacizumab was highly correlated with the change in FLAIR-HI $(r=0.66, P<.05)$. In addition, the relative sensitivity to the change in FLAIR-HI versus RD on treatment was 23.5 times greater for ADC than for RSI-CMs.

\section{DISCUSSION}

Here we demonstrate that RSI, a novel diffusion imaging method, both improves conspicuity within regions of RD relative to ADC and is less influenced by bevacizumab-induced decreases in FLAIR-HI. As shown previously, this effect is because RSI is more sensitive to $\mathrm{RD}$, whereas ADC is more sensitive to hindered diffusion, as seen in areas of FLAIR-HI. In fact, our results suggest that the relative sensitivity of ADC to changes in FLAIR-HI is $>20$ times greater than that derived from RSI-CMs. Thus, RSI-CMs not only facilitate visualization of areas of true RD associated with tumor cellularity (Fig 1), but they should also provide a more robust biomarker of cellularity relative to ADC in the context of treatment-related decreases in FLAIR-HI.

The utility of ADC for detecting high-grade glioma on the basis of increased $\mathrm{RD}$ has been demonstrated in both humans and in animal models. ${ }^{9,11}$ However, concomitant edema and tumor-related necrosis increase ADC values, thereby directly opposing the reduction in $\mathrm{ADC}$ associated with tumor. As a result, it can be difficult to discriminate tumor, edema, and NAWM from one another on ADC maps because $\mathrm{ADC}$ values in tumor may approximate those of NAWM. ${ }^{21-23}$ Indeed, mouse models and clinical studies of patients with high-grade glioma have demonstrated that $\mathrm{ADC}$ is an unreliable 


\section{RSI-CM}
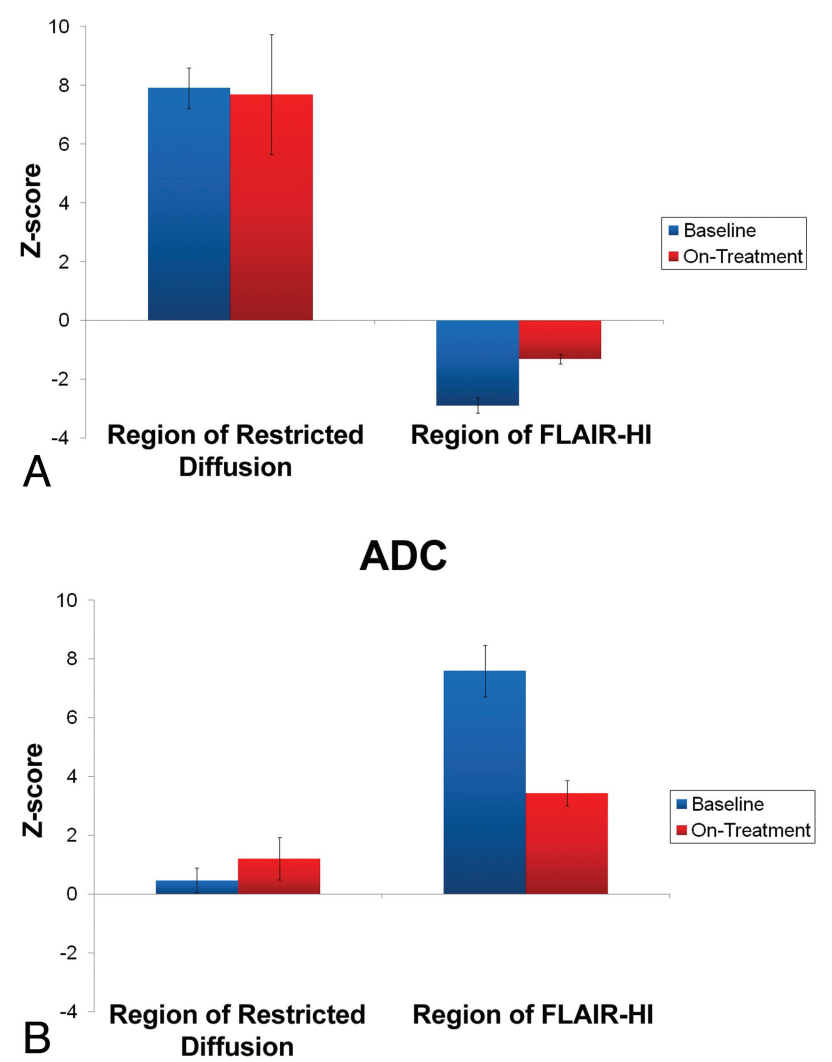

FIG 2. Bar graphs depicting the mean normalized intensity values ( $z$ scores) of the RSI-CMs $(A)$ and the $A D C(B)$ in regions of RD and FLAIR-HI before and on treatment with bevacizumab. Error bars reflect the standard error.

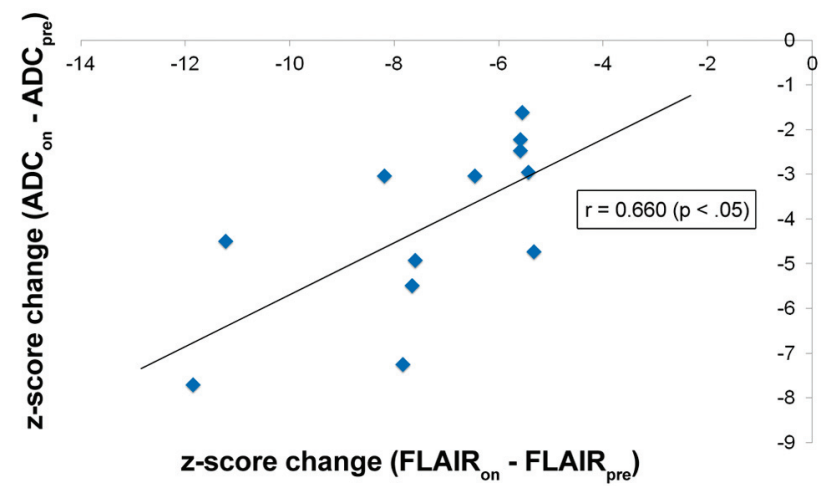

FIG 3. Scatterplot of the relationship between change in ADC $z$ scores (on treatment-pretreatment) and change in FLAIR-HI $z$ scores (on treatment-pretreatment) within the regions of FLAIR-HI. $Z$ scores represent intensity values normalized to NAWM.

tool for differentiating tumor and surrounding edema. ${ }^{1,12,23,24}$ Therefore, when antiangiogenic therapy is administered, subsequent reduction in edema further complicates the interpretation of ADC maps. ${ }^{13,14}$ Although perfusion imaging has been proposed to identify residual or recurrent tumor following antiangiogenic therapy, the results are conflicting. Whereas some studies have shown reduced perfusion following antiangiogenic therapy purportedly due to diminished formation of new blood vessels, ${ }^{25,26}$ other studies have shown elevated perfusion possibly due to vascular normalization. ${ }^{3,27}$
RSI provides a tool to specifically probe the RD compartment in the brain with minimal influence from the hindered diffusion associated with edema or from changes in vasculature attributable to antiangiogenic agents.

One of the limitations to the approach described in this article is that it requires 3D T1-weighted and FLAIR acquisitions and DWI with multiple b-values, by using a specialized pulse sequence that enables estimation and correction of spatial distortions due to B0 field variation. However, we contend that these sequences are necessary to accurately align images across sequences and with time in patients with high-grade gliomas. In this study, we took several steps to improve image registration across sequences and time in our patients. First, we used a method to correct for spatial distortions in EPI data by combining EPI scans acquired with opposite phase-encoding polarities and thus opposite spatial distortion patterns. ${ }^{17}$ Second, we used a nonlinear registration algorithm to improve the alignment of images with time. ${ }^{28,29}$ These methods have been previously applied to serial MRI in large-scale clinical studies ${ }^{29,30}$ and have been shown to have high sensitivity compared with existing methods for measuring brain changes with time. ${ }^{31}$ In addition, our study is limited by a small sample size, heterogeneity of our patient sample, a lack of clinical follow-up, and lack of pathologic confirmation of recurrent tumor in many of our patients. A larger sample size with tissue obtained from targeted biopsies is needed to fully understand the nature of the local RSI signal and to determine its clinical utility. In particular, RSI, like ADC, is not specific to tumor cellularity per se, despite the greater sensitivity of RSI to regions of RD. For example, recent studies have reported regions of marked persistent $\mathrm{RD}$ in certain patients following treatment with bevacizumab that do not appear to reflect aggressive tumor. ${ }^{26,32}$ Although the etiology of these strokelike lesions ${ }^{26}$ on DWI/ADC remains uncertain, pathologic confirmation in a handful of cases has revealed atypical gelatinous necrosis. ${ }^{32}$ RSI, like ADC, is unable to differentiate these regions of RD caused by bevacizumabinduced hypoxia and radiation necrosis from those that reflect increased cellularity due to recurrent tumor. ${ }^{26}$

In our study, pathology reports from 5 patients after initiation of bevacizumab therapy revealed that regions of diffusion restriction detected on RSI-CMs and ADC were indeed positive for recurrent high-grade glioma (Table). However, RD secondary to atypical necrosis that arises from bevacizumab-induced hypoxia cannot be ruled out in the remaining patients. Using other advanced imaging modalities, such as PET and perfusion imaging, may be highly beneficial in disambiguating the true nature of regions of persistent $\mathrm{RD}$. Of note, multispectral imaging analysis incorporating PET and perfusion to further probe these regions of persistent RD would require highly precise image registration. In this context, using methods that correct for spatial distortions within each technique and coregistering images by using a nonlinear algorithm, as described above, become paramount. Applying such a multispectral approach to further interrogate regions of RD seen on RSI-CMs and to better ascertain the specificity of the RSI signal is currently underway in our laboratory.

\section{CONCLUSIONS}

As the clinical landscape continues to evolve for monitoring response to therapy in patients with high-grade gliomas, new imaging techniques will need to be developed that increase the 
sensitivity and specificity to residual/recurrent tumor versus treatment-induced changes. We introduce a new technique, RSI, that increases the sensitivity to areas of RD and is less influenced by changes in FLAIR-HI relative to standard ADC. Future research is needed by using multispectral imaging and targeted biopsies in a large cohort of patients to determine the specificity of RSI to tumor and to evaluate its overall prognostic value.

\section{ACKNOWLEDGMENTS}

We thank patients at the UCSD Moores Cancer Center NeuroOncology Program for their generous participation.

Disclosures: Nathan S. White—RELATED: Support for Travel to Meetings for the Studies or Other Purposes: Holly M. Girard-RELATED: Grant: NIH. * Ajit Shankaranarayanan—UNRELATED: Employment: GE Healthcare. Santosh Kesari—UNRELATED: Consultancy: Merck, Genentech, University of California, Berkeley (UCB), Comments: Speakers Bureau, Grants/Grants Pending: Merck, Genentech, Merck KGaA, Novartis, $\mathrm{NIH}$, Radiation Therapy Oncology Group, Payment for Lectures (including service on Speakers Bureaus): Merck, Genentech, UCB. Carrie R. McDonald—RELATED: Grant: $\mathrm{NIH}$.* Anders M. Dale—RELATED: Grant: NIH, Other: University of California, San Diego (UCSD), ${ }^{*}$ Comments: I am a named inventor on 2 patent applications related to the work under consideration: US8160319, 61483591, UNRELATED: Grants/Grants Pending: $\mathrm{NIH},{ }^{\star}$ Patents (planned, pending, or issued): UCSD, ${ }^{*}$ Massachusetts General Hospital, ${ }^{\star}$ Stock/Stock Options: CorTechs Labs, Comments: I am a founder and hold equity in CorTechs Labs Inc, La Jolla, California, and serve on its scientific advisory board. The terms of this arrangement have been reviewed and approved by the University of California, San Diego, in accordance with its conflict-of-interest policies. *Money paid to the institution.

\section{REFERENCES}

1. Macdonald DR, Cascino TL, Schold SC Jr, et al. Response criteria for phase II studies of supratentorial malignant glioma. J Clin Oncol 1990;8:1277-80

2. Sorensen AG, Batchelor TT, Wen PY, et al. Response criteria for glioma. Nat Clin Pract Oncol 2008;5:634-44

3. Jain RK. Normalization of tumor vasculature: an emerging concept in antiangiogenic therapy. Science 2005;307:58-62

4. Hygino da Cruz LC Jr, Rodriguez I, Domingues RC, et al. Pseudoprogression and pseudoresponse: imaging challenges in the assessment of posttreatment glioma. AJNR Am J Neuroradiol 2011;32:1978-85

5. Chenevert TL, Stegman LD, Taylor JM, et al. Diffusion magnetic resonance imaging: an early surrogate marker of therapeutic efficacy in brain tumors. J Natl Cancer Inst 2000;92:2029-36

6. Pope WB, Kim HJ, Huo J, et al. Recurrent glioblastoma multiforme: ADC histogram analysis predicts response to bevacizumab treatment. Radiology 2009;252:182-89

7. Gerstner ER, Frosch MP, Batchelor TT. Diffusion magnetic resonance imaging detects pathologically confirmed, nonenhancing tumor progression in a patient with recurrent glioblastoma receiving bevacizumab. J Clin Oncol 2010;28:e91-93

8. Jain R, Scarpace LM, Ellika S, et al. Imaging response criteria for recurrent gliomas treated with bevacizumab: role of diffusion weighted imaging as an imaging biomarker. J Neurooncol 2010;96:423-31

9. Sugahara T, Korogi Y, Kochi M, et al. Usefulness of diffusionweighted MRI with echo-planar technique in the evaluation of cellularity in gliomas. J Magn Reson Imaging 1999;9:53-60

10. Chenevert TL, Sundgren PC, Ross BD. Diffusion imaging: insight to cell status and cytoarchitecture. Neuroimaging Clin N Am 2006;16: 619-32, viii-ix

11. Castillo M, Smith JK, Kwock L, et al. Apparent diffusion coefficients in the evaluation of high-grade cerebral gliomas. AJNR Am J Neuroradiol 2001;22:60-64

12. Stadnik TW, Chaskis C, Michotte A, et al. Diffusion-weighted MR imaging of intracerebral masses: comparison with conventional
MR imaging and histologic findings. AJNR Am J Neuroradiol 2001;22:969-76

13. Batchelor TT, Sorensen AG, di Tomaso E, et al. AZD2171, a panVEGF receptor tyrosine kinase inhibitor, normalizes tumor vasculature and alleviates edema in glioblastoma patients. Cancer Cell 2007;11:83-95

14. Pope WB, Lai A, Nghiemphu P, et al. MRI in patients with highgrade gliomas treated with bevacizumab and chemotherapy. Neurology 2006;66:1258-60

15. White NS, Leergaard TB, D'Arceuil H, et al. Probing tissue microstructure with restriction spectrum imaging: histological and theoretical validation. Hum Brain Mapp 2013;34:327-46

16. White NS, McDonald CR, Farid N, et al. Improved conspicuity and delineation of high-grade primary and metastatic brain tumors using "restriction spectrum imaging": quantitative comparison with high b-value DWI and ADC. AJNR Am J Neuroradiol 2013;34: 859-64

17. Holland D, Kuperman JM, Dale AM. Efficient correction of inhomogeneous static magnetic field-induced distortion in echo planar imaging. Neuroimage 2010;50:175-83

18. McDonald CR, White NS, Farid N, et al. Recovery of white matter tracts in regions of peritumoral FLAIR hyperintensity with use of restriction spectrum imaging. AJNR Am J Neuroradiol 2013;34: 1157-63

19. Holodny AI, Makeyev S, Beattie BJ, et al. Apparent diffusion coefficient of glial neoplasms: correlation with fluorodeoxyglucose-positron-emission tomography and gadolinium-enhanced MR imaging. AJNR Am J Neuroradiol 2010;31:1042-48

20. Gerstner ER, Chen PJ, Wen PY, et al. Infiltrative patterns of glioblastoma spread detected via diffusion MRI after treatment with cediranib. Neuro Oncol 2010;12:466-72

21. Yamasaki F, Kurisu K, Satoh K, et al. Apparent diffusion coefficient of human brain tumors at MR imaging. Radiology 2005;235:985-91

22. Maier SE, Sun Y, Mulkern RV. Diffusion imaging of brain tumors. NMR Biomed 2010;23:849-64

23. Eis M, Els T, Hoehn-Berlage M. High resolution quantitative relaxation and diffusion MRI of three different experimental brain tumors in rat. Magn Reson Med 1995;34:835-44

24. Brunberg JA, Chenevert TL, McKeever PE, et al. In vivo MR determination of water diffusion coefficients and diffusion anisotropy: correlation with structural alteration in gliomas of the cerebral hemispheres. AJNR Am J Neuroradiol 1995;16:361-71

25. Keunen O, Johansson M, Oudin A, et al. Anti-VEGF treatment reduces blood supply and increases tumor cell invasion in glioblastoma. Proc Natl Acad Sci U S A 2011;108:3749-54

26. Rieger J, Bahr O, Muller K, et al. Bevacizumab-induced diffusionrestricted lesions in malignant glioma patients. J Neurooncol 2010;99:49-56

27. Sorensen AG, Emblem KE, Polaskova P, et al. Increased survival of glioblastoma patients who respond to antiangiogenic therapy with elevated blood perfusion. Cancer Res 2012;72:402-07

28. Jovicich J, Czanner S, Greve D, et al. Reliability in multi-site structural MRI studies: effects of gradient non-linearity correction on phantom and human data. Neuroimage 2006;30:436-43

29. Holland D, Dale AM. Nonlinear registration of longitudinal images and measurement of change in regions of interest. Med Image Anal 2011;15:489-97

30. McDonald CR, Hagler DJ Jr, Girard HM, et al. Changes in fiber tract integrity and visual fields after anterior temporal lobectomy. $\mathrm{Neu}$ rology 2010;75:1631-38

31. Holland D, McEvoy LK, Dale AM. Unbiased comparison of sample size estimates from longitudinal structural measures in ADNI. Hum Brain Mapp 2012;33:2586-602

32. Mong S, Ellingson BM, Nghiemphu PL, et al. Persistent diffusionrestricted lesions in bevacizumab-treated malignant gliomas are associated with improved survival compared with matched controls. AJNR Am J Neuroradiol 2012;33:1763-70 